\title{
How a Non-hierarchical Neutrino Mass Matrix Can Arise
}

\author{
S.M. Barr \\ Bartol Research Institute \\ University of Delaware \\ Newark, DE 19716
}

\begin{abstract}
One puzzle of neutrino masses and mixings is that they do not exhibit the kind of strong "hierarchy" that is found for the quarks and charged leptons. Neutrino mass ratios and mixing angles are not small. A possible reason for this is proposed here. It is based on the fact that typical realistic grand unified models contain particles with unification-scale masses which, when integrated out, can yield a neutrino mass matrix that is not of the standard seesaw form.
\end{abstract}

In the standard (or type I) see-saw mechanism [1], the observed lefthanded neutrinos derive their masses from their Dirac couplings to righthanded neutrinos. These right-handed neutrinos have Majorana masses that in grand unified theories (GUTs) are expected to be of order the unified scale $M_{G U T}$. When the right-handed neutrinos are integrated out, an effective $d=5$ operator is induced that produces a mass matrix for the left-handed neutrinos given by the well-known "see-saw formula":

$$
M_{\nu}=-M_{N} M_{R}^{-1} M_{N}^{T}
$$

Here $M_{N}$ is the Dirac mass matrix of the neutrinos, whose elements are less than or of order the Weak scale. $M_{R}$ is the Majorana mass matrix of the right-handed neutrinos. And $M_{\nu}$ is the effective mass matrix of the light left-handed neutrinos. 
In GUTs based on $S O(10)$ or related groups, there is typically a close connection between the Dirac mass matrix of the neutrinos $M_{N}$ and the (Dirac) mass matrices of the up quarks, down quarks, and charged leptons $\left(M_{U}, M_{D}\right.$, and $M_{L}$ respectively). For instance, in minimal $S O(10)$ these matrices are all proportional. The small interfamily mass ratios and small CKM angles suggest that these other mass matrices are "hierarchical" in structure (i.e. their entries decrease as one goes up and to the left in the matrix, in the usual convention). This would lead us to expect a hierarchical structure also for $M_{N}$. In that case, the see-saw formula would make $M_{\nu}$ hierarchical unless there were a "Dirac-Majorana conspiracy" [2], i.e. unless there were hierarchies in both $M_{N}$ and $M_{R}$ that practically cancelled each other out in $M_{\nu}$. Such a conspiracy is not easy to achieve in a natural way.

On the other hand, the experimental evidence suggests that the light neutrinos have at most a very weak hierarchy. The solar mixing angle and atmospheric mixing angle are large, and the ratio of neutrino masses $m_{2} / m_{3}$ is not very small either, about $\frac{1}{6}$ judging from the fact that $\delta m_{\text {atm }}^{2} \sim 3 \times 10^{-3}$ $\mathrm{eV}$ [3] and $\delta m_{\text {atm }}^{2} \sim 7.3 \times 10^{-5} \mathrm{eV}$ [4]. Thus we have a problem: if $M_{\nu}$ is given by the standard see-saw formula, why doesn't it exhibit a strong interfamily hierarchy? In this letter we suggest a possible solution.

In realistic grand unified models, it is typical that there are many fermions with masses of order $M_{G U T}$ besides the three right-handed neutrinos. When integrated out, these other superheavy fermions can give effective higherdimension Yukawa operators that contribute to the masses of the observed quarks and leptons. In fact, in many models, this is precisely the way in which the quarks and leptons of the lighter families get their small masses, i.e. the way the interfamily mass hierarchies arise. (See $[5,6]$ for examples). The crucial point for the present discussion is that these superheavy fermion multiplets typically contain neutrino-like states; and these, when integrated out, give contributions to the masses of the observed light neutrinos that are not necessarily of the standard (or "type I") see-saw form shown in Eq. (1). These non-type-I contributions to $M_{\nu}$ do not involve the effective Dirac neutrino mass matrix $M_{N}$ and consequently there is no reason why they have to be hierarchical.

The possibility of non-type-I contributions to $M_{\nu}$ arising from integrating out superheavy fermion multiplets in grand unified models was analyzed in a general way in Ref. [7]. Here we present a concrete model that illustrates in a transparent way how a non-hierarchical $M_{\nu}$ can arise from these contributions 
even when family symmetry causes $M_{N}, M_{U}, M_{D}$, and $M_{L}$ to be hierarchical.

Consider an $S O(10)$ model in which, in addition to the three families (contained in three spinor multiplets, denoted $\mathbf{1 6}_{i}, i=1,2,3$ ), there are three family-antifamily pairs, denoted $\mathbf{1 6}{ }_{i}^{\prime}, \overline{\mathbf{1 6}}_{i}^{\prime}, i=1,2,3$. There is a vector multiplet of Higgs fields, denoted $\mathbf{1 0} \mathbf{0}_{H}$, which contains the MSSM doublets of Higgs, $H_{u}$ and $H_{d}$. There are three Higgs multiplets denoted $\Omega_{i}, i=1,2,3$, which contain components that are neutral under the Standard Model gauge group, have $B=L=0$, and obtain $O\left(M_{G U T}\right)$ vacuum expectation values (VEVs). These will break a $U(1)$ family symmetry and play the role of "familons" or "Froggatt-Nielson fields" [5].

Under the family symmetry, called $U(1)_{F}$, the multiplets just itemized have the following charges. The $\mathbf{1 6}_{i}$ and $\overline{\mathbf{1 6}}_{i}^{\prime}$ have charge $q_{i}$, and the $\mathbf{1 6}{ }_{i}^{\prime}$ has charge $-q_{i}$, where $q_{1}=y, q_{2}=z$, and $q_{3}=0$. The Higgs multiplet $\mathbf{1 0}_{H}$ is neutral under $U(1)_{F}$, and the Froggatt-Nielson fields $\Omega_{1}, \Omega_{2}$, and $\Omega_{3}$ have charges $(-y-z),-z$, and 0 , respectively.

These assignments allow the following renormalizable terms to appear in the Yukawa part of the superpotential.

$$
\begin{aligned}
W_{\text {Yukawa }} & =\sum_{i} M_{i}\left(\overline{\mathbf{1 6}}_{i}^{\prime} \mathbf{1 6} \mathbf{6}_{i}^{\prime}\right)+\left(\overline{\mathbf{1 6}}_{3}^{\prime} \mathbf{1 6} \mathbf{6}_{3}^{\prime}\right) \Omega_{3} \\
& +\sum_{i}\left(\mathbf{1 6}_{i} \mathbf{1 6} \mathbf{6}_{i}^{\prime}\right) \mathbf{1 0}+\left(\mathbf{1 6}_{3} \mathbf{1 6}\right) \mathbf{1 0}_{H}+\left(\mathbf{1 6}_{3}^{\prime} \mathbf{1 6} \mathbf{6}_{3}^{\prime}\right) \mathbf{1 0}_{H} \\
& +\left(\overline{\mathbf{1 6}}_{3}^{\prime} \mathbf{1 6} \mathbf{6}_{2}+\overline{\mathbf{1 6}}_{2}^{\prime} \mathbf{1 6} \mathbf{6}_{3}\right) \Omega_{2}+\left(\overline{\mathbf{1 6}}_{2}^{\prime} \mathbf{1 6} \mathbf{6}_{1}+\overline{\mathbf{1 6}}_{1}^{\prime} \mathbf{1 6} \mathbf{6}_{2}\right) \Omega_{1}+\left(\overline{\mathbf{1 6}}_{3}^{\prime} \mathbf{1 6} \mathbf{6}_{3}\right) \Omega_{3} .
\end{aligned}
$$

We do not show the dimensionless Yukawa couplings, which we assume all to be $O(1)$. Note that the explicit mass terms and the couplings of the $\mathbf{1 0}_{H}$ are both flavor-diagonal. The non-trivial flavor structure in the model comes from the couplings of $\Omega_{i}$

In addition to all of these multiplets, we require there to be Higgs that break $B-L$ at the GUT scale. We take these to be in several $\overline{\mathbf{1 2 6}}_{H}$ and $\mathbf{1 2 6} \mathbf{6}_{H}$ multiplets. (There are reasons, including not having the unified coupling blow up as it runs from $M_{G U T}$ to $M_{P \ell}$, that it might be more realistic to assume that $B-L$ is broken by spinors of Higgs fields. However, for simplicity of exposition, we assume that it is broken by $\overline{\mathbf{1 2 6}}_{H}$ and $\mathbf{1 2 6}_{H}$. It would be quite straightforward to modify the model to replace the $\overline{\mathbf{1 2 6}}_{H}$ and $\mathbf{1 2 6} \mathbf{6}_{H}$ by spinor Higgs.) The mass matrix $M_{R}$ of the right-handed neutrinos comes from terms of the form $\left(\mathbf{1 6}_{i} \mathbf{1 6}_{j}\right) \overline{\mathbf{1 2 6}}_{H}$. We will also assume that a mass matrix $\bar{M}_{R}^{\prime}$ arises 
from terms of the form $\left(\overline{\mathbf{1 6}}_{i}^{\prime} \overline{\mathbf{1 6}}_{j}^{\prime}\right) \mathbf{1 2 6}_{H}$. The $\overline{\mathbf{1 2 6}}_{H}$ and $\mathbf{1 2 6}_{H}$ multiplets will have whatever $U(1)_{F}$ charges are required to allow these couplings.

The family hierarchy is assumed to arise from a hierarchy in the VEVs of the familons, with

$$
\Omega_{1} \ll \Omega_{2} \ll \Omega_{3} .
$$

Before considering neutrino masses, let us see how the hierarchies in $M_{U}$, $M_{D}$, and $M_{L}$ arise. For specificity, let us consider $M_{L}$. The multiplets $\mathbf{1 6}_{i}$, $\mathbf{1 6}_{i}^{\prime}$, and $\overline{\mathbf{1 6}}_{i}^{\prime}$ contain charged leptons that we will denote $\left(\ell_{i}^{+}, \ell_{i}^{-}\right),\left(\ell_{i}^{+\prime}, \ell_{i}^{-\prime}\right)$, and $\left({\overline{\ell^{+}}}_{i}^{\prime},{\overline{\ell^{-}}}_{i}^{\prime}\right)$, respectively. If the mixing of the $\mathbf{1 6}_{i}$ and $\mathbf{1 6}_{i}^{\prime}$ is small, then it is approximately the case that the light leptons are contained in the "unprimed multiplets" $\mathbf{1 6}_{i}$ while the superheavy leptons are contained in the "primed multiplets" $\overline{\mathbf{1 6}}_{i}^{\prime}$ and $\mathbf{1 6}_{i}^{\prime}$. There is a $d=4$ operator that contributes directly to $M_{L}$, namely $\left(\ell_{3}^{-} \ell_{3}^{+}\right) H_{d}$, coming from the term $\left(\mathbf{1 6}_{3} \mathbf{1 6}_{3}\right) \mathbf{1 0}_{H}$ in Eq. (2). In addition, $M_{L}$ receives contributions from integrating out the superheavy (primed) leptons, as follows.

The terms in Eq. (2) include leptons mass terms of the form

$$
\begin{aligned}
W_{\text {lepton }} & \supset \sum_{i j} M_{i j}\left({\overline{\ell^{-}}}_{i}^{\prime} \ell_{j}^{-\prime}+{\overline{\ell^{+}}}_{i}^{\prime} \ell_{j}^{+\prime}\right) \\
& +\sum_{i j}\left(M_{\Omega}\right)_{i j}\left({\overline{\ell^{-}}}_{i}^{\prime} \ell_{j}^{-}+{\overline{\ell^{+}}}_{i}^{\prime} \ell_{j}^{+}\right) \\
& +\sum_{i j}\left(m_{H}\right)_{i j}\left(\ell_{i}^{-} \ell_{j}^{+\prime}+\ell_{i}^{-} \ell_{j}^{+\prime}\right) .
\end{aligned}
$$

where these matrices have the forms

$M=\left(\begin{array}{ccc}M_{1} & 0 & 0 \\ 0 & M_{2} & 0 \\ 0 & 0 & M_{3}\end{array}\right), M_{\Omega}=\left(\begin{array}{ccc}0 & \omega_{1} & 0 \\ \omega_{1} & 0 & \omega_{2} \\ 0 & \omega_{2} & \omega_{3}\end{array}\right), m_{H}=\left(\begin{array}{ccc}m_{1} & 0 & 0 \\ 0 & m_{2} & 0 \\ 0 & 0 & m_{3}\end{array}\right)$

The matrices $M$ and $M_{\Omega}$ have elements of $O\left(M_{G U T}\right)$, while the elements of $m_{H}$ are of order the weak scale. And $\omega_{1} \ll \omega_{2} \ll \omega_{3}, M_{1} \sim M_{2} \sim M_{3}$, $m_{1} \sim m_{2} \sim m_{3}$. When the superheavy, primed leptons are integrated out, the diagrams of Fig. 1 lead to a contribution to $M_{L}$ of the form

$$
\begin{aligned}
\delta M_{L} & =m_{H} M^{-1} M_{\Omega}+\text { Transpose } \\
& =\left(\begin{array}{ccc}
0 & \bar{\omega}_{1} & 0 \\
\bar{\omega}_{1} & 0 & \bar{\omega}_{2} \\
0 & \bar{\omega}_{2} & \bar{\omega}_{3}
\end{array}\right)
\end{aligned}
$$


where $\bar{\omega}_{1}=\omega_{1}\left(\frac{m_{1}}{M_{1}}+\frac{m_{2}}{M_{2}}\right), \bar{\omega}_{2}=\omega_{2}\left(\frac{m_{2}}{M_{2}}+\frac{m_{3}}{M_{3}}\right)$, and $\bar{\omega}_{3}=\omega_{3}\left(2 \frac{m_{3}}{M_{3}}\right)$. Note the hierarchical form of $\delta M_{L}$. The $d=4$ operator $\ell_{3}^{-} \ell_{3}^{+} H_{d}$ just gives a contribution to the 33 element that is of the same order as the 33 element of $\delta M_{L}$.

Since the same types of diagrams give the mass matrices $M_{U}, M_{D}$, and $M_{N}$, these matrices are all related. In fact, if the fields $\Omega_{i}$ were $S O(10)$ singlets, these matrices would not "know" that $S O(10)$ is broken, and one would have the minimal $S O(10)$ relations $M_{L}=M_{D} \propto M_{U}=M_{N}$. If, on the other hand, some of the $\Omega_{i}$ are adjoints or other non-singlets, then Clebsch coefficients can appear that distinguish the matrices of fermions of different types (i.e. of different Standard Model quantum numbers).

The Dirac mass matrix $M_{N}$ thus arises both from the direct $d=4$ term $\left(\nu_{3} N_{3}^{c}\right) H_{u}$ (from $\left(\mathbf{1 6}_{3} \mathbf{1 6}_{3}\right) \mathbf{1 0}_{H}$ ) and from the diagrams of Fig. 2. Like $M_{L}$, it is hierarchical in form. The right-handed neutrinos $N_{i}^{c}$ get superlarge Majorana mass matrix $\left(M_{R}\right)_{i j}\left(N_{i}^{c} N_{j}^{c}\right)$ from terms of the form $\left(\mathbf{1 6}_{i} \mathbf{1 6}_{j}\right) \overline{\mathbf{1 2 6}}_{H}$. Consequently, one has the type I see-saw contribution $M_{\nu}^{\text {type-I }}=-M_{N} M_{R}^{-1} M_{N}^{T}$, which arises from graphs of the kind shown in Fig. 3. However, these are not the only kind of graphs that can contribute to $M_{\nu}$. If there are terms of the form $\left(\overline{\mathbf{1 6}}_{i}^{\prime} \overline{\mathbf{1 6}}_{j}^{\prime}\right) \mathbf{1 2 6}_{H}$, then a simpler graph, shown in Fig. 4, contributes. Basically, this graph "short circuits" the usual type-I diagrams by eliminating the standard right-handed neutrinos $N_{i}^{c}$ as intermediate particles. To put it another way, it "eliminates the middle-man". This graph gives

$$
M_{\nu}^{\text {non-type }-I}=m_{H} M^{-1 T} \bar{M}_{R}^{\prime} M^{-1} m_{H}^{T} .
$$

Note that the graph in Fig. 4 does not contain insertions of the VEVs of the Froggatt-Nielson fields $\Omega_{i}$, and therefore does not "know" about the family hierarchy. Consequently, this non-type-I contribution to $M_{\nu}$ has no reason to be hierarchical and will not in general be so. Moreover, since these non-typeI contributions do not depend on the small parameters $\Omega_{1} / \Omega_{3}$ and $\Omega_{2} / \Omega_{3}$, they will dominate over the type-I contributions, except perhaps for the 33 element where they should be comparable. One expects, therefore, neutrino mixing angles and neutrino mass ratios that are of order one, as is indeed observed.

It should be noted that if the term $\left(\mathbf{1 6}_{i} \mathbf{1 6}_{j}\right) \overline{\mathbf{1 2 6}}_{H}$ does not exist, i.e. the matrix $M_{R}=0$, then the form of $M_{\nu}$ is not well approximated by Eq. (7). The diagrams can be misleading, and one must really do the matrix 
diagonalization to find out the form of $M_{\nu}$. However, if both $M_{R}$ and $\bar{M}_{R}^{\prime}$ are non-zero and $M_{R}$ is non-singular, then $M_{\nu}$ is given aproximately by the sum of Eq. (1) and Eq.(7).

One might ask whether the even simpler kind of diagram shown in Fig. 5 might give a non-type-I, hierarchical contribution to $M_{\nu}$ if there were terms of the form $\left(\mathbf{1 6}^{\prime} \mathbf{1 6}\right) \overline{\mathbf{1 2 6}}_{H}$. It would appear so from the structure of the diagram; but it turns out that no non-type-I contribution of this kind arises unless there is also a contribution of the kind given in Fig. 4 and Eq. (7). This is not obvious from inspection of the diagrams, but can be shown by looking in detail at the full neutrino mass matrix diagonalization problem, as was done in the general analysis of Ref. [7].

In conclusion, we see that in realistic GUT models there can be additional contributions to $M_{\nu}$ that are not of the type I form, and that these contributions can be non-hierarchical even if a family symmetry enforces a hierarchical pattern for all the Dirac mass matrices, that of the neutrinos $\left(M_{N}\right)$ and those of the up quarks, down quarks, and charged leptons $\left(M_{U}\right.$, $M_{D}, M_{L}$ ). This is due to the fact that there typically exist in realistic GUT models superheavy neutrino-like fermions besides the three right-handed neutrinos $N^{c}$ usually taken into account. Integrating out these other states can induce contributions to $M_{\nu}$ that do not involve the $N^{c}$ at all, and thus do not depend on the form of the Dirac neutrino mass matrix $M_{N}$. Finally, it should be mentioned that a recent paper of Nir and Shadmi [8] has interesting points of contact with the present work. For a discussion of the similarities and differences of their approach and the ideas on which the present work is based, see the discussion at the end of [7].

\section{References}

[1] M. Gell-Mann, P. Ramond, and R. Slansky, in Supergravity, Proceedings of the Workshop. Stony Brook, New York, 1979, ed. P. van Nieuwenhuizen and D.Z. Freedman (North-Holland, Amsterdam, 1979), p. 315; T. Yanagida, in Proc. Workshop on Unified Theory and the Baryon Number of the Universe, Tsukuba, Japan, 1979, ed. O. Sawada and A. Sugramoto (KEK Report No. 79-18, Tsukuba, 1979); R.N. Mohapatra and G. Senjanovic, Phys. Rev. Lett. 44, 912 (1980); 
S.L. Glashow in Quarks and leptons, Cargese (July 9-29, 1979), ed. M. Levy et al. (Plenum, New York, 1980), p. 707.

[2] S.M. Barr and I. Dorsner, Nucl. Phys. B585, 79 (2000).

[3] Y. Ashie et al., (Super-Kamiokande Collaboration), hep-ex/0404034;

E. Kearns (Super-Kamiokande Collaboration), talk given at Neutrino2004.

[4] T. Araki et al., (KamLAND Collaboration) hep-ex/0406035; G. Gratta (KamLAND Collaboration), talk given at Neutrino2004; J.N. Bahcall and C. Pena-Garay, JHEP 0311:004 (2003).

[5] C.D. Froggatt and H.B. Nielson, Nucl. Phys. B147, 277 (1979).

[6] S.M. Barr, Phys. Rev. D21, 1424 (1980); R. Barbieri and D.V. Nanopoulos, Phys. Lett. B95, 43 (1980); S.M. Barr, Phys. Rev. D24, 1895 (1981); Phys. Rev. D42, 3150 (1990); G. Anderson, S. Dimopoulos, L.J. Hall, S. Raby, and G.D. Starkman, Phys. Rev. D49, 3660 (1994); C.H. Albright and S.M. Barr, Phys. Rev.; C.H. Albright, K.S. Babu, and S.M. Barr, Phys. Rev. Lett. 81, 1167 (1998); K.S. Babu, J. Pati, and F. Wilczek, Nucl. Phys. B566, 33 (2000).

[7] S.M. Barr and B. Kyae, Phys. Rev. D70, 075005 (2004).

[8] Y. Nir and Y. Shadmi, JHEP 0411:055 (2004). 


\section{Figure Captions}

Fig. 1: A contribution to the charged lepton mass matrix $M_{L}$ coming from integrating out the $O\left(M_{G U T}\right)$ charged leptons in the "primed" multiplets (denoted by the double lines). The coupling to the familons $\Omega$ gives the family hierarchy.

Fig. 2: A contribution to the neutrino Dirac mass $M_{N}$ coming from integrating out the $O\left(M_{G U T}\right)$ neutrinos in the "primed" multiplets (denoted by double lines). These diagrams are related by $S O(10)$ symmetry to those in Fig. 1.

Fig. 3: A typical standard (or type I) seesaw contribution to $M_{\nu}$. The double lines represent the $O\left(M_{G U T}\right)$ fermions that are integrated out to generate $M_{N}$. (See Fig. 2.)

Fig. 4: A contribution to $M_{\nu}$ that is not of the standard type I form. It does not involve the standard right-handed neutrinos $N_{i}^{c}$ of the three families. It also does not involve the familon field $\Omega$ and is not therefore hierarchical in structure.

Fig. 5: A diagram that seems to give a non-type-I contribution to $M_{\nu}$ but generally does not. 


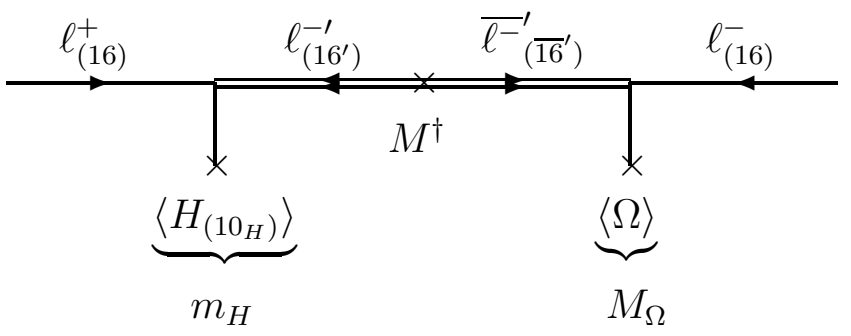

Fig. 1(a)

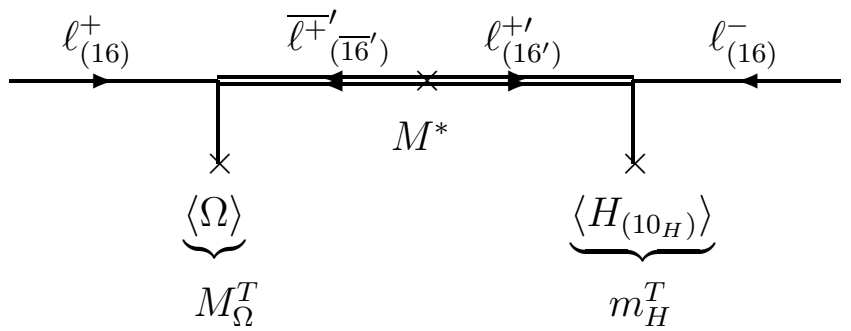

Fig. 1(b) 


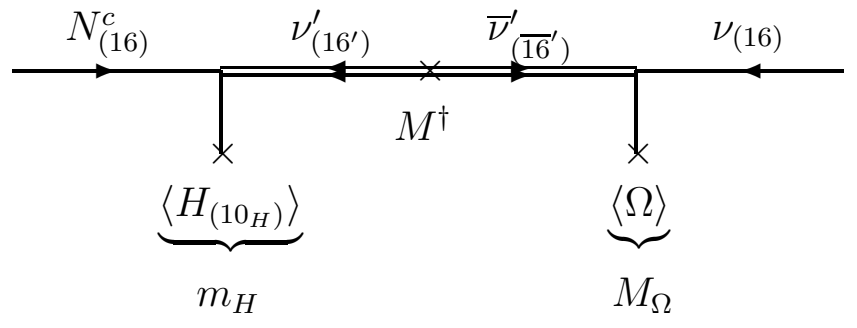

Fig. 2(a)

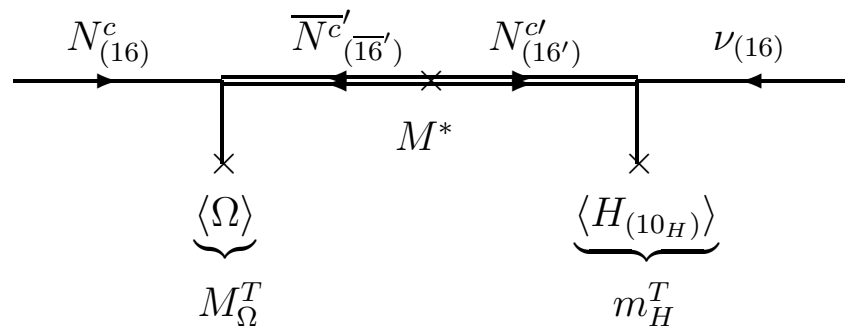

Fig. 2(b) 


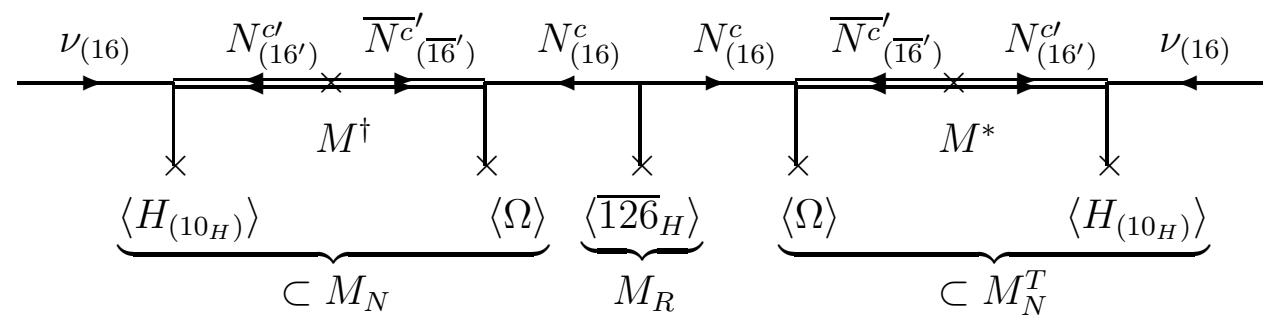

Fig. 3

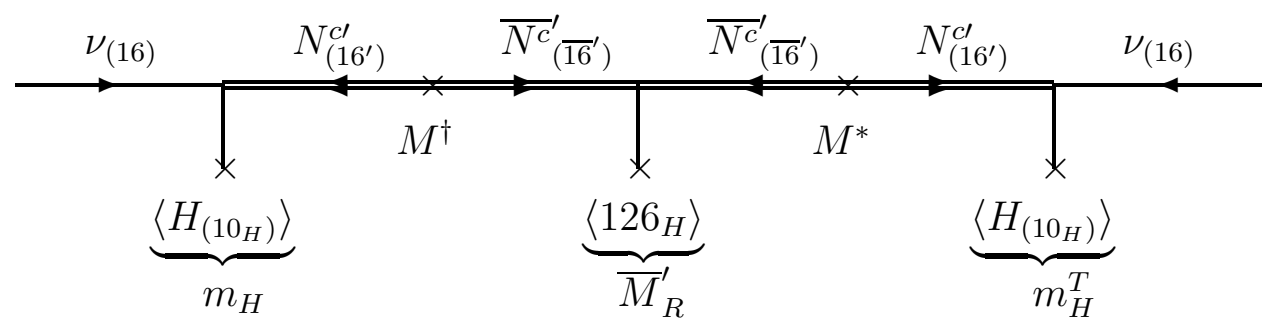

Fig. 4 


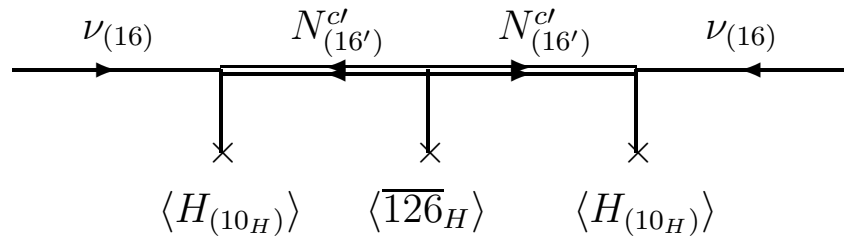

Fig. 5 